\title{
Modelado paramétrico y fabricación digital para la concepción de edificios de museo ambientalmente adecuados para el clima subtropical húmedo de Brasil.
}

Parametric modeling and digital manufacturing for the conception of museum buildings environmentally suitable for the subtropical wetland climate of Brazil.

\author{
Laline Cenci \\ UNILA, Brasil \\ lalinecenci@gmail.com \\ Rodreigo Garcia Alvarado \\ UBB, Chile \\ rgarcia@ubb.cl
}

\begin{abstract}
Museums exhibit a growing development in the world, promoting buildings with significant expressions. Nevertheless, the relation of the building and its environmental performance is fundamental in the conception of new buildings. The problem originates in the early stages of design, where it is not possible to evaluate it environmentally. The methodology uses three art museum buildings in the humid subtropical climate of Brazil, whose geometries are completely different. After analyzing and relating its performance to its environmental and geometric characteristics a parametric modeling tool is proposed and the digital manufacture as a product of the process has been carried out.
\end{abstract}

Keywords: Parametric Modeling; Digital Manufacturing; Art museums; Environmental Compatibility; Subtropical Humid Climate of Brazil.

\section{Introducción}

Este artículo presenta una experiencia relacionada a la relación del diseño paramétrico y la fabricación aplicada a la concepción de edificios de museos de arte. Presenta datos relevantes para el ámbito de la arquitectura de museos, la cual se presenta con una variedad de geometrías complejas conformando las imágenes de las ciudades. También se propone el uso de las herramientas digitales y la fabricación digital para etapas tempranas del proceso de proyecto. Para ello, se ha realizado un workshop de diseño paramétrico y fabricación digital para la conformación de edificios de museos de arte en el clima subtropical húmedo de Brasil, en la Universidad Comunitaria de la Región de Chapecó (UNOCHAPECÓ)-Santa Catarina, Brasil.

De acuerdo con el estudio del Instituto Brasileiro de Museus (IBRAM, 2013), Brasil inició el siglo XX con 12 museos, y ahora cuenta con 3200 entidades. Se ha evaluado que en 2009 los museos brasileños fueron visitados por cerca de 82 millones de personas y, de los 5.564 municipios brasileños, un $21,1 \%$ (1.172) poseen, al menos, un museo. La meta del Gobierno es que la mitad de las ciudades brasileñas tenga por lo menos un museo hacia el 2020 , lo que implicaría más de 2.000 nuevos museos en el país, con más de 250 millones de visitantes al año, la mayor parte localizados en climas

\footnotetext{
${ }^{1}$ Un parámetro es un término constante o variable en una función que determina su forma específica. Una ecuación paramétrica generalmente relaciona dos o más variables.
}

subtropicales. Estas consideraciones motivan el estudio del diseño y gestión del ambiente interior de los nuevos edificios, especialmente en estas zonas.

La creciente demanda por la cultura, en conjunto con mejores recintos expositivos, incentiva que las personas se encuentren, una cantidad relevante de tiempo, en un ambiente donde se exhiben elementos significativos: libros, esculturas, fotografías, documentos, pinturas, entre otros. Para asegurar el buen funcionamiento de un museo es fundamental mantener sus colecciones dentro de valores ambientales adecuados para su preservación y garantizar, al mismo tiempo, que las condiciones de confort sean las apropiadas para los visitantes. No obstante, una evaluación ambiental de tres estudios de caso significativos en esta zona se ha llevado a cabo y los resultados fueron incorporados en una herramienta de modelado paramétrico.

\section{Diseño Paramétrico y Fabricación Digital}

Con origen en las matemáticas, el término paramétrico refiere a un valor o medida sobre la cual algo depende generalmente representado por una variable que puede ser alterada. $1 \mathrm{EI}$ concepto de paramétrico en el diseño ha sido asociado a la noción de variación del diseño, específicamente en el desarrollo de la representación computacional de un objeto y 
su modelado geométrico (Mitchell, 1994; Mc Cullough y Mitchell, 1996).

Los sistemas paramétricos se presentan como una nueva alternativa donde se combinan conceptos de diseño y programación para ser adaptados al campo del diseño, buscando explorar las posibilidades existentes en torno al desarrollo de una idea geométrica y para construir una nueva forma de pensar los problemas en este campo.

Actualmente existe un sinnúmero de herramientas de modelado tridimensional que le permiten al diseñador representar geometrías en una pantalla. Sin embargo, estas cuentan con limitantes que no permiten que el proceso de modificación de la misma pueda ser efectuado de forma rápida y fácil. El diseño paramétrico ofrece la posibilidad de encontrar nuevas maneras de originar soluciones y de controlar el proceso de diseño. De esta manera, se produce tanto una automatización respecto a la definición de las geometrías, así como en la generación de un modelo que posibilita cambios y/o ediciones al diseño de manera ágil y significativa (Tedeschi, 2011). La modelación paramétrica, también conocida por el concepto de modelado por restricción, introduce un cambio en el proceso de diseño, relacionando y alterando informaciones involucradas de manera sistematizada (Woodbury, 2010). El diseño paramétrico funciona como un apoyo en el proceso de diseño y ha sido ampliamente utilizada en la arquitectura en los últimos años como un soporte frente al uso de herramientas computacionales (Singh, 2011). Ante ello, en la arquitectura, el uso de parametrización ha permitido un ir y venir entre la práctica del diseño y la evaluación del impacto de esas decisiones durante la etapa de diseño.

Asimismo estas herramientas pueden ser poderosas para el estudio del desempeño ambiental de los edificios (Caldas \& Norford, 2002) porque permiten controlar definiciones de la forma arquitectónica. Aris, et al., (2006) muestran cómo el desarrollo de un diseño paramétrico es capaz de optimizar las aperturas de un espacio para conseguir el consumo energético mínimo y después diseñar alternativas de solución de posibles fachadas. Por otro lado Caldas (2002) hace uso del diseño paramétrico para minimizar el uso de aire acondicionado, electricidad y costos de la arquitectura. Otros autores usan los algoritmos genéticos en el proceso de diseño creativo para la generación de geometrías de edificios (Marin, et al., 2008).

El uso del diseño paramétrico en la arquitectura permite diversas aplicaciones. Turrin et al., $(2011,2012)$ presentan los beneficios derivados de combinar el modelos paramétrico y los algoritmos genéticos, para lograr el rendimiento de una solución geométrica en relación a las ganancias solares y luz natural con cubiertas. Wang et al., (2007) hacen una aplicación del diseño paramétrico para la solución de fachadas con rendimiento en confort térmico y ahorro energético. Se observa una tendencia que ha sido motivada actualmente por grandes fuentes teóricas y nuevas tecnologías, fortaleciendo así, cuestiones relacionadas a la resiliencia de la arquitectura y de las ciudades.
Según Tramontano (2011) los sistemas paramétricos interesan principalmente al proceso en arquitectura, primero porque automatizan la relación de los valores atribuidos, propiciando instantáneamente, en la tela de la computadora un imagen gráfica correspondiente a la ecuación utilizada e también porque proporcionan alterar los valores de ellas adecuando automáticamente estas modificaciones.

Para la complejidad de las formas arquitectónicas contemporáneas, fluidas, como en los casos de edificios de museos de arte el diseño paramétrico se presenta indispensable. OURCIUOLI (2009) y PUPO (2008) recomiendan la utilización de herramientas paramétricas, para proyectos de alta complejidad constructiva y tectónica, pues estos exigen software que comporten la modelaje en automatizando el proceso de fabricación y, en el caso de la Fabricación Digital, el producto es enviado directamente a una impresión laser, CNC o impresora 3D. En cuanto que los programas CAD (Computer Aidded Design) son utilizados apenas para la representación gráfica.

Investigaciones académicas como Massachusetts Institute of Technology, Architectural Association, la Escuela Superior de Arquitectura de la Universidad Internacional da Catalunya, Ball State University, Swiss Federal Institute os Techology, Universidade Técnica de Lisboa, y Delft University of Technology muestran a través de estudios exploratorios la importancia de la arquitectura asociada a informática, programación computacional con utilización de softwares parametricos y scripts (OURCIUOLI, 2009 y PUPO, 2008).

\section{Objetivos}

El estudio objetiva la implementación de algoritmos en una plataforma de diseño paramétrica basada en los estudios de caso, para la concepción de edificios ambientalmente compatibles. Es posible decir que estos sistemas se componen de mecanismos relativamente simples que consiguen asociar y entregar de forma rápida resultados en torno a la manipulación e integración de diferentes criterios de diseño y la fabricación digital del modelo constituye como elemento fundamental indisociable del proceso de proyecto. De esta manera, el diseño puede llegar a ser concebido como una búsqueda óptima y apropiada para asistir a la Compatibilidad Ambiental en los edificios.

\section{Los casos de estudio}

Los tres casos de estudio son edificios recientes y significativos, construidos para ser museos y pertenecen al clima subtropical húmedo de Brasil, zona en el cual se encuentra la mayor parte de los museos en Brasil (65\%). De este porcentaje, se eligieron las tres ciudades que cuentan con la mayor cantidad de museos: São Paulo, Curitiba y Porto Alegre, seleccionando un caso de cada ciudad.

\section{1 - Museu Brasileiro de la Escultura (MUBE-Figura1)}

Localizado en São Paulo (-23 $32^{\prime} 51^{\prime \prime} \mathrm{S} /-46^{\circ} 38^{\prime} 10^{\prime \prime}$ W) y diseñado por el arquitecto Paulo Mendes da Rocha, el edificio, inaugurado en 1994, ocupa un área de 7 mil metros cuadrados. Su terreno triangular, formado por la confluencia 
de dos vías importantes, da espacio a un ejemplo destacado de la arquitectura moderna brasileña. Una gran viga perpendicular enfrenta una de las vías, con un vano de 60 metros que enmarca el paisaje, dejando las salas de exhibición bajo el nivel de la calle. Las tres salas están conectadas $\mathrm{y}$, eventualmente, pueden ser cerradas para adaptarse a distintos tipos de muestra. Las áreas de auditorio, administración y apoyo técnico se encuentran tangentes a las salas de exposición. La condición enterrada del proyecto permite una menor ganancia térmica y, por lo tanto, un menor consumo energético para conservar sus obras. Sin embargo, como el sistema de aire acondicionado atiende a las tres salas, aunque funcionen independientemente, se mantiene sin aire acondicionado en gran parte del tiempo.

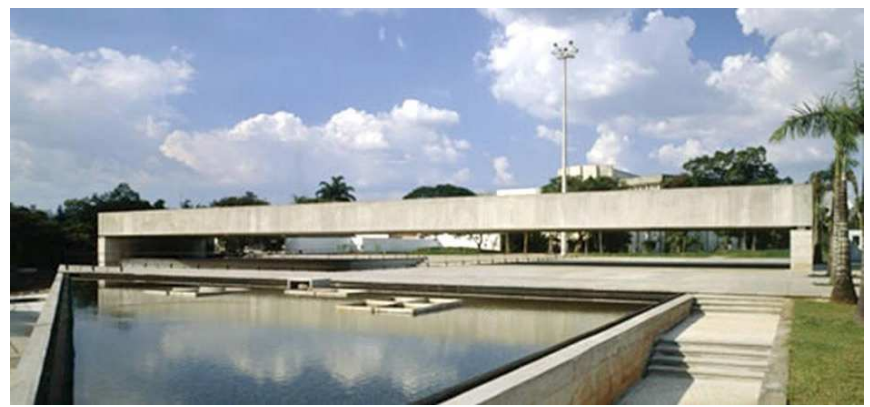

Figura 1. Museo Brasileño de la Escultura

\section{2 - Museu Oscar Niemeyer (MON-Figura 2)}

Localizado en Curitiba (-25 $31^{\prime}$ 52" S/-49 10'32” W), está instalado frente al edificio histórico adaptado para museo e internamente conectado por un túnel. Este edificio de 30 metros de altura, conocido como el "ojo", fue construido en el año 2002 y es uno de los ejemplos de arquitectura brasileña contemporánea. Su salón principal, ubicado en la parte convexa de la estructura, destina cerca de 1,6 mil metros cuadrados para exposiciones, cuyo punto más alto alcanza 12 metros. En su opuesto, el lado cóncavo, está ubicado el área de apoyo: auditorio y sala de reuniones. Según la institución, para mantener su clima interior, dentro de las normas de conservación de las obras y confort de sus visitantes, el consumo energético del edificio es muy alto ( $\sin$ aire acondicionado, en su interior, se ha llegado a medir $55^{\circ} \mathrm{C}$ ).

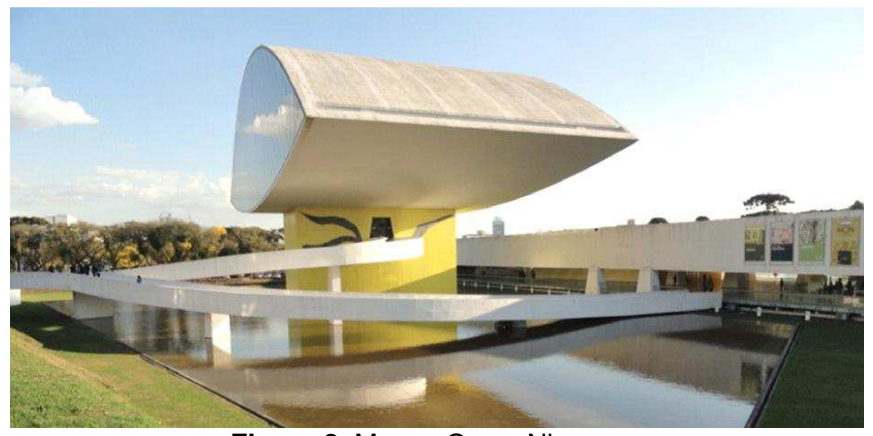

Figura 2. Museo Oscar Niemeyer

\section{3 - Fundação Iberê Camargo (FIC-Figura 3)}

Inaugurado en 2008 , en Porto Alegre (3001' 59"S/- 51 ${ }^{\circ} 13^{\prime}$ 48 " W), el proyecto fue realizado por el arquitecto portugués Álvaro Siza Vieira. Está emplazado en la tangente de la trama urbana, frente al lago Guaíba, y en la estrecha porción plana que queda al costado de un cerro de baja altura. En la parte más profunda, está posicionado el volumen principal con una planta baja y 3 pisos, destinados a las salas de exposiciones interconectadas que, configuradas en forma de " $L$ ", generan un atrio de 21 metros de altura. Todas las áreas de apoyo (acervo, auditorio, biblioteca, talleres, salas para cursos y los servicios públicos) están dispuestas en el subsuelo. Para minimizar la excavación, el edificio fue levantado a 1,4 metros de la cota cero del terreno. El visitante advierte una sucesión de pequeños volúmenes hasta la gran masa de cuatro pisos. Internamente, ocultas en sus paredes internas, están ubicadas las instalaciones eléctricas y también aperturas en los guardapolvos y cornisas para las entradas y salidas de aire. La temperatura y humedad interna de cada recinto del edificio son monitoreadas las 24 horas del día. El sistema de aire acondicionado enfría por la noche, cuando el costo de la energía eléctrica es más barato, para refrigerar el ambiente durante el día, reduciendo los costos de la operación y aumentando la utilización de la energía. Según la institución, el edificio consume del $30 \%$ al $40 \%$ menos de energía que una construcción convencional.

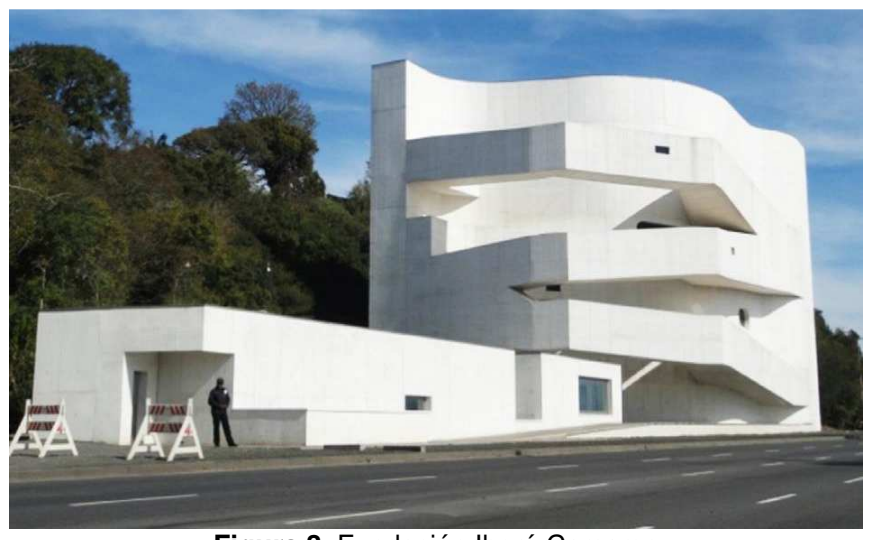

Figura 3. Fundación Iberé Camargo

En la Tabla 1 se presentan sus características implementadas al modelado paramétrico.

Tabla 1. Características de los casos y parámetros geométricos. Elaboración de la autora.

\begin{tabular}{|l|c|c|c|}
\hline Parámetro & MUBE & MON & FIC \\
\hline Área Proyección $\left(\mathrm{m}^{2}\right)$ & 3071,34 & 2100 & 849 \\
\hline Área total $\left(\mathrm{m}^{2}\right)$ & 3071,34 & 2934,5 & 2450 \\
\hline Longitudinal máx. x $(\mathrm{m})$ & 85 & 70 & 45 \\
\hline Longitudinal máx. y $(\mathrm{m})$ & 55 & 30 & 22 \\
\hline Altura máx. z $(\mathrm{m})$ & 5 & 30 & 25 \\
\hline Número de Pisos & 1 & 2 & 4 \\
\hline Atrio & - & - & $1 / 3$ \\
\hline
\end{tabular}

\section{Metodologia}

La herramienta utilizada para la implementación de una plataforma de diseño está incorporada con el sistema de programación paramétrica Grasshopper® sobre la herramienta de modelado tridimensional Rhinoceros $\AA^{\circledR}$. Según 
Turrin, et al., (2011) existen grandes beneficios derivados de combinar el modelo paramétrico y los algoritmos en etapas tempranas del diseño ya que permite obtener rápidamente gran cantidad de soluciones a un problema mediante la exploración de distintas geometrías.

En el workshop realizado em maio del 2016, en la UNOCHAPECO, los participantes pudieron experimentar, algunos por primera vez, iniciar el proceso de proyecto con una herramienta paramétrica. El workshop fue dividido en tres etapas, una teórica y dos prácticas. En la etapa teórica, se presentó a los participantes la estructura de trabajo, la línea base para el proyecto de museo de arte en el clima subtropical húmedo y las condiciones de diseño para la compatibilidad ambiental. En seguida el primero ejercicio práctico consistía en generar propuestas de geometría con la herramienta paramétrica y en la tercera y última etapa, la fabricación digital. El ejercicio propuesto con la herramienta llevo a los participantes a testear varias propuestas de forma, acompañando simultáneamente sus valores de compacidad, porosidad, esbeltez. También, se podría verificar la cantidad de área de exposición y número de pisos. Para iniciar la generación de la geometría, el usuario elige las dimensiones $(\mathrm{x}, \mathrm{y}, \mathrm{z})$ de un volumen general que sean las proporciones del edificio, esto en los sliders en la caja azul en números enteros dentro de los rangos máximos definidos. Luego, aplicase deformaciones en los sliders del grupo lila, verificando que se cumplan los valores de esbeltez $(0,04)$ y compacidad $(0,5)$ que promueven Compatibilidad Ambiental. A continuación es posible apreciar la interface de trabajo en la Figura 4:

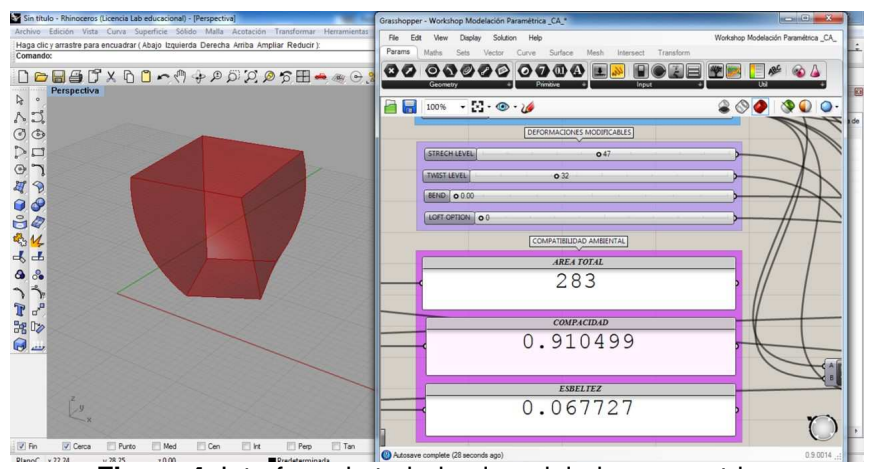

Figura 4. Interface de trabajo el modelado parametrico.

Las variaciones ejecutadas en los sliders de la interface de Grasshopper®, causan efectos en la geometría que es visualizada en Rhinoceros ${ }^{\circledR}$ en la pantalla compartida. Esto, permite un análisis visual de la forma arquitectónica desde distintos puntos de vista para considerar aspectos de localización, funcionalidad y expresividad. Cuando en la propuesta geométrica generada y los valores de compacidad y esbeltez fueron alcanzados, puede exportar el volumen para desarrollarlo más detalladamente o almacenar opciones para comparar.

Definidos los criterios geométricos y sus transformaciones tridimensionales: taper, twist, stretch y bend, tal como en el trabajo de Marin (2008), se definieron el dominio de criterios ambientales o evaluación de la compatibilidad ambiental. Para las características ambientales de la geometría a ser generada, se vincularon las ecuaciones de compacidad, de porosidad, de perforación y de esbeltez, ya que según Serra y Coch (1995) estas son las características que definen la forma. Estos valores son resultantes de la geometría generada en donde, datos como la superficie global, volumen y alturas son revisados a medida que se aplican las transformaciones, permitiendo simultáneamente vincular la geometría concebida con sus cualidades geométricas asociadas a la compatibilidad ambiental. Con respecto a las ecuaciones que evalúan la compatibilidad de las relaciones de Porosidad $(\mathrm{P})$ ha sido considerada como la diferencia entre la magnitud global del volumen (la media entre ancho y largo, $(\mathrm{X} 1-\mathrm{Y} 1) / 2)$ y la magnitud de espacio abierto interior $(\mathrm{X} 2-\mathrm{Y} 2) / 2)$ estableciendo como valor de referencia (1) el $50 \%$, o sea $\mathrm{P}=$ $(\mathrm{X} 1-\mathrm{Y} 1) / 2) \times$ 0,5-(X2-Y2)/2). Para Compatibilidad Ambiental de museos en esta zona el valor preferente es $P<0,25$ (un patio reducido para evitar superficie envolvente) y el óptimo $\mathrm{P}=0$ (sin patio). La herramienta paramétrica se programó para partir con volúmenes integrales, al evaluar directamente debería validar con porosidad óptima (0). Así, los valores de esbeltez y compacidad son sensibles a las modificaciones de la geometría sin considerar pátios.

Finalmente, se llevó a cabo la fabricación digital de los modelos generados con la herramienta. Fuero realizados con o software 123D make de Autodesk $®$ y materializados con la cortadora a laser del Laboratorio Pronto $3 \mathrm{D}$ de la UNOCHAPECÓ.

\section{Resultados}

En la Figura 5, a continuación se presentan las distintas geometrías que fueron elaboradas con el auxilio de la herramienta de modelado paramétrico y posteriormente fabricadas digitalmente. Es posible apreciar las distintas posibilidades de diseño del museo que obedecen los criterios de Compatibilidad Ambiental insertados en la herramienta de diseño.

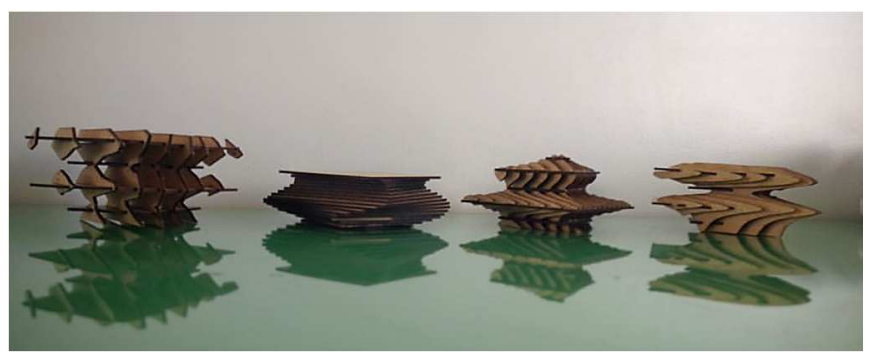

Figura 5.Modelos Fabricados en el workshop.

\section{Conclusiones}

La herramienta presentada sigue una tendencia contemporánea donde la computación empieza a participar del proceso de diseño ya en las etapas tempranas del proyecto. Una herramienta computacional es importante para optimizar el trabajo y facilitar la incorporación de criterios comprobables, más que una respuesta formal gratuita. Con respecto a la herramienta paramétrica desarrollada, se concluye que en los aspectos relacionados a las geometrías 
forman parte del proceso de diseño, otorgando a los arquitectos criterios que promuevan un bajo consumo energético a estos edificios. Los software de simulación energética disponibles no facilitan el modelado de geometrías exentas con la aplicabilidad de variables ambientales o para alcanzar un mínimo de Compatibilidad Ambiental, al menos no a nivel de anteproyecto. Esta herramienta, a pesar de bastante primitiva debido a su restricción formal asociada a los casos de estudios es considerada apropiada para el trabajo regular de un arquitecto, con una formación y uso de herramientas computacionales habituales, durante el proceso de proyecto y la Fabricación Digital es un importante aliado en este proceso. Por fin, se concluye que, estudios de compatibilidad ambiental en edificios de esta categoría ciertamente puede traer muchos beneficios y por eso deben ser realizados. La integración de criterios de diseño que apoyan la concepción de nuevos proyectos para la calidad ambiental ha sido llevada a cabo a la vez que puso en discusión el tema.

\section{Agradecimientos}

A cada uno de los participantes del whorkshop, Laboratorio Pronto 3D de Chapecó, UNOCHAPECÓ, Universidad del Biobío, MON/PR, MUBE/SP, FIC/RS.

\section{Referencias}

ARIS, et al. (2006).Energy conscious automated design of building facades using genetic algorithms In Communicating Space(s), eCAADe06 Proceedings.eCAADe.Pag.898-903.

CALDAS, L. et. al. An evolutionary model for sustainable design. Management of Environmental Quality. Vol. 14(3)383-397/ 2003.DOI: $10.1108 / 14777830310479450$.

CALDAS, L. G., Norford, L. K. (1999). Genetic Algorithms tool for optimization.

IBRAM. Instituto Brasileiro de Museus. Disponible en: http://www.ibram.gov.br/

MARIN, Phillipe; BIGNON, Jean-Claude; LEQUAY, Hervé. 2008. Paramètres environnementaux et mécanismes de conception évolutionnaire. Disponible en [http://halshs.archivesouvertes.fr/halshs-00440272]

MITCHELL, William J; MCCULLOUCH, M. (1995). Design Digital Media. Ed.John Wiley \& Sons. Canada.
(2008). A lógica da Arquitetura. Traducción de Gabriela Celani. Editora USP. São Paulo.

SERRA, Rafael; COCH, Helena (1995). Arquitectura y energía natural. Universidad Politécnica de Cataluña. Barcelona, España

KOLAREVIC, B. Designing and manufacturing architecture in the digital age. Architectural information management, 2001.

OLIVEIRA, M. R; FABRICIO, M.M. Prototipagem rápida como ferramenta de projeto e ensino de arquitetura- visita a laboratórios de prototipagem. Disponible en: http://www.iau.usp.br/pesquisa/grupos/arquitec/Marina/Artigo_Pr ojetar.pdf.

ORCIUOLI, A. Arquitetura Digital - TI Aplicada à Arquitetura: o Antes e o Depois. Revista aU, edição 181, abril 2009.

PUPO, R. Inserção da PROTOTIPAGEM e FABRICAÇÃO DIGITAIS no processo de projeto: um novo desafio para o ensino de Arquitetura. Tese (Doutorado) Engenharia Civil - Faculdade de Engenharia Civil, Universidade Estadual de Campinas, 2009.

SINGH, V. \&Gu, N. 2012. Towards an integrated generative design framework.DesignStudies. Volume 33, Issue 2.185-207. Disponible en: http://www.sciencedirect.com/science/article/pii/S0142694X1100 0391

TEDESCHI.A. (2011).Parametric Architecture with Grasshopper. Brienza, Italia. Edizione Le Penser.

TURRIN et.al. Desing Explorations of performance driven geometry in architectural design using parametric model and genetic algorithms. Advanced Engineering Informatics.V.25, 656$675 / 2011$.

2012.Performative skins for passive climatic comfort. A parametric design

TRAMONTANO, M. (2011). Quando pesquisa e ensino se conectam: design paramétrico, fabricação digital e projeto de arquitetura. Disponible en: http://pdf.blucher.com.br.s3-sa-east1.amazonaws.com/designproceedings/sigradi2015/100144.pdf .

WANG, et al. Corrigendum to Facade design optimization for naturally ventilated residential buildings' in Singapore. Energy and Buildings. Volume 39-2007.Issue 8.954-961.

WOODBURY, Robert. (2010). Elements of Parametric Design. Routledge, New York, USA. ISBN-10: 0415779871| ISBN13: $978-04157798760$ 